\title{
121.
}

\section{NOTE ON A QUESTION IN THE THEORY OF PROBABILITIES.}

[From the Philosophical Magazine, vol. vi. (1853), p. 259.]

The following question was suggested to me, either by some of Prof. Boole's memoirs on the subject of probabilities, or in conversation with him, I forget which; it seems to me a good instance of the class of questions to which it belongs.

Given the probability $\alpha$ that a cause $A$ will act, and the probability $p$ that $A$ acting the effect will happen; also the probability $\beta$ that a cause $B$ will act, and the probability $q$ that $B$ acting the effect will happen; required the total probability of the effect.

As an instance of the precise case contemplated, take the following: say a day is called windy if there is at least $w$ of wind, and a day is called rainy if there is at least $r$ of rain, and a day is called stormy if there is at least $W$ of wind, or if there is at least $R$ of rain. The day may therefore be stormy because of there being at least $W$ of wind, or because of there being at least $R$ of rain, or on both accounts; but if there is less than $W$ of wind and less than $R$ of rain, the day will not be stormy. Then $\alpha$ is the probability that a day chosen at random will be windy, $p$ the probability that a windy day chosen at random will be stormy, $\beta$ the probability that a day chosen at random will be rainy, $q$ the probability that a rainy day chosen at random will be stormy. The quantities $\lambda, \mu$ introduced in the solution of the question mean in this particular instance, $\lambda$ the probability that a windy day chosen at random will be stormy by reason of the quantity of wind, or in other words, that there will be at least $W$ of wind; $\mu$ the probability that a rainy day chosen at random will be stormy by reason of the quantity of rain, or in other words, that there will be at least $R$ of rain. 
The sense of the terms being clearly understood, the problem presents of course no difficulty. Let $\lambda$ be the probability that the cause $A$ acting will act efficaciously; $\mu$ the probability that the cause $B$ acting will act efficaciously; then

$$
\begin{aligned}
& p=\lambda+(1-\lambda) \mu \beta, \\
& q=\mu+(1-\mu) \alpha \lambda,
\end{aligned}
$$

which determine $\lambda, \mu$; and the total probability $\rho$ of the effect is given by

$$
\rho=\lambda \alpha+\mu \beta-\lambda \mu \alpha \beta
$$

suppose, for instance, $\alpha=1$, then

$$
p=\lambda+(1-\lambda) \mu \beta, \quad q=\mu+\lambda-\lambda \mu, \quad \rho=\lambda+\mu \beta-\lambda \mu \beta,
$$

that is, $\rho=p$, for $p$ is in this case the probability that (acting a cause which is certain to act) the effect will happen, or what is the same thing, $p$ is the probability that the effect will happen.

Machynlleth, August 16, 1853. 